Review

\title{
BENEFICIAL EFFECTS OF SILYMARIN AGAINST NEPHROTOXICITY CAUSED BY SOME CHEMICALS
}

\author{
V. Ivanov ${ }^{1 *}$, V. Slavova ${ }^{1}$, D. Georgieva ${ }^{1}$, V. Petrova-Tacheva ${ }^{2}$, A. Tolekova $^{3}$ \\ ${ }^{1}$ Department of Neurology, Psychiatry and MDS, Faculty of Medicine, Trakia University, \\ Stara Zagora, Bulgaria \\ ${ }^{2}$ Department of Molecular Biology, Immunology and Medical Genetics, Faculty of Medicine, \\ Trakia University, Stara Zagora, Bulgaria \\ ${ }^{3}$ Department of Physiology, Pathophysiology and Pharmacology, Faculty of Medicine, \\ Trakia University, Stara Zagora, Bulgaria
}

\begin{abstract}
Silybum marianum (L) Gaertn (Carduusmarianus L., Asteraceae) seeds have been used as a traditional remedy for liver and gallbladder diseases, and has been especially valued for its properties against hepatitis and cirrhosis for centuries. Publications on its beneficial effects in the treatment of diabetes, on its radio-protective effects, and properties against chemical weapons intoxication have proliferated in the medical literature over the last few years. Silymarin is used as a chemoprotective and anticancer agent. It also has protective properties against the toxic effects of some drugs used in oncology, as well as antibiotics, which was the topic of the first part of our review on the nephroprotective effects of Silymarin. The purpose of this publication is to summarise the results of our research into the effects of Silymarin for reducing the toxic damage caused by chemical agents like fungi toxins, organic solvents, heavy metals, etc.
\end{abstract}

Key words: silymarin, silibin, silibinin, nephrotoxicity

\section{INTRODUCTION}

The kidneys quite often suffer damage caused by different toxic compounds like fungal poisons, heavy metals or organic solvents. Silymarin has been used for many years in alternative and modern medicine to treat liver diseases. The antioxidant, anti-inflammatory and anti-apoptotic properties of silymarin have made it an interesting herb for medicine and have suggested that it can be used as a potential renoprotective agent. Silymarin may exhibit significant protective effects against different toxic compounds. Whether the preventive application of silymarin can serve as an effective strategy to prevent druginduced nephrotoxicity is yet to be proven by clinical trials (1).

Silymarin is a standardized extract of Silybum marianum (Blessed milk thistle). It's chemical

\footnotetext{
*Correspondence to: Veselin Ivanov, Department of Neurology, Psychiatry and MDS, Faculty of Medicine, Trakia University, Stara Zagora, Bulgaria,veskoasenov@abv.bg
}

content mainly reveals silybin, dehydrosylibin (DHSB), quercetin, taxipholine, silicrystine and a number of other compounds, all of which are known to have a number of beneficial health effects. There is evidence that they can play a part in slowing down tumor growth, preventing liver toxicity and protecting a number of organs against ischemic damage. Silymarin's hepatoprotective effect of preventing $\alpha$-amanitin and alcohol intoxication in particular, which causes damage to the liver, is well-recognised (2). There is also strong evidence that silymarin has antibacterial (3) and anti-cancirogenic effects (4). Xanthine oxidase is an enzyme involved in tissue oxidative damage following ischemiareperfusion. Dehydrogenase/oxidase ratio in rats' kidney homogenates decreases during ischemia and reperfusion. Silymarin contains two flavonoids, quercetin and silybin, characterized as free radical scavengers that produce the protective effect by preventing the dehydrogenase/oxidase ratio from decreasing during ischemia-reperfusion (5). 
Use of silymarin for reducing nephrotoxicity caused be medicaments

Sylimarin can effectively reduce the toxic effects a number of medicaments like paracetamol, antibiotics, anti-tuberculosis and anticancer agents, as well as immunosuppressants can have on the kidneys. We have already published an article on that topic, so we are not referring to these issues here (6).

\section{Nephroprotective effects of Silymarin in mushroom poisoning}

Some of the most severe and often deadly mushroom poisoning is caused by amatoxins contained in 35 different species belonging to three genera: Amanita, Galerina and Lepiota. The major amatoxins, alpha-, beta- and gamma-amanitines are bicyclic octapeptide derivatives that damage the liver and the kidney by irreversibly binding to RNA polymerase II (7). In such situations, the outcome is often lethal. The reason most often is renal damage in addition to the extremely serious damage the toxins cause to the liver. Animal studies have shown that silymarin is able to counteract this liver damage caused by alpha-amanitin. The fact, that alpha-amanitine causes pathological changes in the kidney and that these lesions can be almost completely prevented by pre-treatment of rats with silymarin has now been largely demonstrated by biochemical and histological techniques by Vogel et al., (8). Aflatoxin $\mathrm{B}_{1}\left(\mathrm{AFB}_{1}\right)$ is a potent hepatotoxic and hepatocarcinogenic mycotoxin. The mechanism of cellular damage caused by $\mathrm{AFB}_{1}$ is not fully understood. Lipid peroxidation is one of the main manifestations of oxidative damage and has been shown to play an important role in the toxicity and carcinogenesis of many carcinogens. In a study of Rastogi et al., it was found that there was increased lipid peroxidation in the kidney of a rat, induced by aflatoxin $B_{1}(2 \mathrm{mg} / \mathrm{kg}$, ip), while treatment with silymarin returned it to near normal levels (9). Moreover, Trakulsrichai et al. (10) analyzed 55 cases of mushroom poisoning in Thailand between May 2013 and August 2015 (amatoxins). The mortality rate was $27.3 \%$. In 8 of the cases, the patients received silymarin in large doses. Only one of those patients died (12.5\%). Due to the small number of cases, the efficacy of orally administered silymarin cannot be confirmed. In this analysis no adverse effects of the high dose of silymarin have been reported. Therefore, more studies are needed to clarify the efficacy of this medicine. It is known that after a long latency period (3-17 days), the nephrotoxins in poisonous Cortinariusrubellus and Cortinariusorellanus fungi can cause severe damage to the epithelium of the proximal renal tubule. Svendsen et al. (11) describe 5 cases of intoxication after ingestion of such mushrooms. Their toxicity leads to persistent renal failure in four of the five patients, with no specific treatment available. Therapy targets renal failure, including dialysis and possible transplantation. It is also known that the Amanita virosa fungus causes serious toxic damage to the liver and the kidney after an initial asymptomatic latent phase. The same authors describe a case in which a patient who ingested Amanita virosa developed hepatotoxicity and nephrotoxicity. He received silibinin and symptomatic treatment and recovered. The authors conclude that the intoxication due to toxins contained in Cortinarius rubellus, Cortinarius orellanus and Amanita virosa fungi can be treated with a non-specific antidote silibinin.

Fumonisin $\mathrm{B}_{1}$ is a mycotoxin commonly contained in maize. It is known to have hepatotoxic and nephrotoxic effects on domestic and experimental animals and causes leukoencephalomalisation in horses and pulmonary edema in pigs. It is a potent inhibitor of ceramide synthase. Inhibition results in the accumulation of free sphingoid bases in cells and tissues. In swine epithelial cells (LLC-PK1), fumonisin $\mathrm{B}_{1}$ induces increased expression of tumor necrosis factor alpha (TNF alpha), regardless of the accumulation of sphingoid bases. In a study conducted by $\mathrm{He}$ et al., pharmacological approaches for interfering with fumonisin $\mathrm{B}_{1}$ toxicity were investigated using the LLC-PK1 cell model. The fumonisin $\mathrm{B}_{1}$ toxicity was analyzed using cell viability and lactate dehydrogenase release. Pre-treatment with silymarin prevented the effects induced by fumonisin $\mathrm{B}_{1}$ on cellular viability and the release of lactate dehydrogenase. When silymarin was added 6 or 24 hours after treatment of cells with fumonisin $B_{1}$, the reduced cell viability was restored and the increased release of lactate dehydrogenase was suppressed. Study results indicate that silymarin potentially prevents fumonisin $\mathrm{B}_{1^{-}}$ induced toxicity by modulating TNF-alpha 
expression or signals after ceramide synthase inhibition (12).

\section{Silymarin's effect on tetrachloromethane, thioacetamide, pyridine and bromobenzene poisoning}

Abdel-Kader et al. (13) investigated the protective effect of silymarin on the kidneys against tetrachloromethane- histopathologic and biochemical intoxication by observing lactate dehydrogenase (LDH), creatinine, creatine kinase levels, urea and uric acid. Groups of cells treated with silymarin showed almost normal functions with mild toxic signs. The roots, bark, stem/twigs, and leave of Fraxinus xanthoxyloids are used regionally to treat malaria, jaundice, internal injuries, pneumonia, pain, rheumatism, and also in bone fractures. The aim of Younis et al. (14), was to evaluate the antioxidant capacity of methanol extract of $\mathrm{F}$. xanthoxyloides leaves against carbon tetrachloride $\left(\mathrm{CCl}_{4}\right)$ oxidative stress in the kidney of Sprague-Dawley rats. The duration of this experiment was 30 days and doses were given on alternate days. Rat urine was tested for renal function and renal tissue for antioxidant enzymes activity, biochemical markers, comet analysis and histopathology.

Increased urine creatinine, urobilinogen levels and decreased creatinine clearance, protein content and albumin levels were measured and analysed after administration of $\mathrm{CCl}_{4}$ when compared to controls. $\mathrm{CCl}_{4}$ treatment was found to decrease the level of reduced glutathione, catalase, superoxide dismutase, peroxidase, glutathione s-transferase, and glutathione reductase and tissue proteins, while increasing the levels of thiobarbituric acid, DNA damage, and the level of $\mathrm{H}_{2} \mathrm{O}_{2}$ cells in kidney tissue of experimental animals. Cotreatment with methanol extract of $\mathrm{F}$. xanthoxyloides and silymarin resulted in the restoration of all the above-mentioned renal parameters (14). Thioacetamide is a welldocumented liver toxin that requires oxidative bioactivation to induce its hepatotoxic effect, which ultimately alters amine-lipids and proteins. Ghost et al. [15] conducted a mouse treatment study to investigate whether silymarin could protect the thioacetamideinduced oxidative stress leading to hepatic and renal damage. The results show that thioacetamide generates reactive oxygen species (ROS), causes oxidative stress, and induces apoptosis in the liver and kidney cells through JNK as well as PKC and MAPKs signalling. However, all these harmful effects of thioacetamide can be suppressed by silymarin, which not only is a ROS scavenger but also induces a PI3K-Akt cell survival pathway in the liver and prevents apoptotic pathways in both organs. Histological studies, collagen staining and DNA fragmentation analysis also support these results. Silymarin has a favorable role against thioacetamidemediated liver and renal pathophysiology. Cengiz (16) conducted a similar study on twenty-eight rats divided into four groups. Group 1 was used as a control (saline $0.5 \mathrm{ml}$ intraperitoneally (i.p.)). Group 2 was given 50 $\mathrm{mg} / \mathrm{kg}$ thioacetamide (i.p.). Group 3 was administered thioacetamide after $50 \mathrm{mg} / \mathrm{kg}$ silymarin (per os (p.o.)). Group 4 was treated with thioacetamide after $100 \mathrm{mg} / \mathrm{kg}$ silymarin. At the end of the fourteenth day of the study, tissue and blood samples from the animals were collected for morphological and biochemical analysis. The results showed that silymarin treatment had a beneficial effect on the toxic effects in rats. In addition, $100 \mathrm{mg} / \mathrm{kg}$ silymarin revealed better protection than 50 $\mathrm{mg} / \mathrm{kg}$, which was demonstrated by histopathological and biochemical tests. In conclusion, treatment with silymarin prior to thioacetamide may protect the kidney tissues against thioacetamide-induced nephrotoxicity. Tunca et al. (17) conducted experiments to evaluate silymarin protection against the toxic effects of pyridine on the kidney of experimental animals. To Syrian hamsters were intraperitoneally injected with $400 \mathrm{mg} / \mathrm{kg}$ dose of pyridines. Another group was injected with the same amount of pyridine and was given $200 \mathrm{mg} / \mathrm{kg}$ of silymarin daily, for 4 days. Pyridine induced diffuse degeneration and necrosis of proximal and distal renal tubular cells. Administration of silymarin significantly reduced the toxic effects.

\section{Silymarin and intoxication caused by heavy metals}

Heavy metal intoxication can damage the kidneys. Chronic heavy metal intoxication is caused relatively frequently under poor working conditions and occupational hazards. The most common are lead intoxication. Alcaraz-Contreras et al. (18) conducted experiments with Wistar rats $(200 \pm 20)$ treated with $2000 \mathrm{ppm}$ lead acetate in drinking water for 8 weeks. Toxic changes in the kidney were reported. Administration of silymarin (100 $\mathrm{mg} / \mathrm{kg}$ ) for 8 weeks provided significant protection against the toxic effect of $\mathrm{Pb}$ on the 
kidneys. In similar experiments with rats as well, El-Khishin et al. (19) found a positive effect of silymarin on the toxic effect of lead on the kidneys. The difference was that, in addition to silymarin, the rats were given garlic extract. Chtourou et al. (20) administered manganese orally, with drinking water $(20 \mathrm{mg}$ $\left.\mathrm{MnCl}_{2} / \mathrm{ml}\right)$ with or without silymarin (100 $\mathrm{mg} / \mathrm{kg}$ intraperitoneally) for 30 days. The data show that silymarin significantly prevents Mninduced nephrotoxicity, indicated by both the renal impairment diagnostic parameters such as urea, uric acid and electrolyte levels in the urine, and by histopathological analysis. In addition, manganese induces an increase in ROS production and alters the levels of biomarkers associated with oxidative stress in renal tissue. This is evidenced by the increase in lipid peroxidation, protein carbonylation, DNA fragmentation and urinary hydrogen peroxide, while glutathione levels decrease. Silymarin treatment reduces changes in kidney and urine markers, decreases lipid peroxidation markers, and reduces Mn-induced damage. All these changes are supported by histopathological observations. In the study of Navaneethan and Rasool (21) rats received cadmium chloride (3 $\mathrm{mg} / \mathrm{kgb}$.Wt) subcutaneously once a day, for 3 weeks while another group received the same amount of cadmium chloride and silymarin (50 $\mathrm{mg} / \mathrm{kgb}$. Wt), once a day orally, for a period of 3 weeks. At the end of the experiment, the animals were sacrificed and blood and kidney samples were collected. The results of this study reveal the fact that levels of lipid peroxidation, lysosomal enzymes, glycoprotein, cadmium and metallothionein were high in rats treated with cadmium chloride alone and their antioxidant status was reduced compared to the control group. Levels of renal functional markers (urea, uric acid, and creatinine) have been found to be abnormal in serum and urine of rats treated with cadmium chloride alone. On the other hand, the administration of silymarin together with cadmium chloride significantly protected the biochemical changes observed in rats treated with cadmium chloride alone, as demonstrated by histopathology. Thus, oral administration of silymarin significantly protects cadmium-induced nephrotoxicity in rats. In experiments with rats conducted by Prabu and Muthumani (22) animals received oral sodium arsenite $\left(\mathrm{NaAsO}_{2}\right), 5 \mathrm{mg} / \mathrm{kg}$ per day for 4 weeks. This induces significant renal impairment, as evidenced by increased levels of serum urea, uric acid, creatinine with a significant <0.05) decrease in creatinine clearance. As well as significantly reducing the levels of urea, uric acid and creatinine in the urine. Significantly increased levels of lipid peroxidation markers and protein carbonyl content with a significant $(\mathrm{p}<0.05)$ decrease in non-enzymatic antioxidants (total sulfhydryl groups, reduced glutathione, vitamin $\mathrm{C}$ and vitamin E) and enzyme antioxidants (superoxide dismutase, catalase, glutathione peroxidase and glutathione S-transferase, glutathione reductase) and membrane-bound ATPase. Co-administration of silybinin (75 $\mathrm{mg} / \mathrm{kg}$ per day) with as resulted in a reversible effect of arsenic-induced biochemical changes in the kidneys, accompanied by a significant decrease in lipid peroxidation and an increase in the level of the renal antioxidant defense system. Histopathological and immunohistochemical studies in rat kidney also showed that silibinin $(75 \mathrm{mg} / \mathrm{kg}$ per day) significantly reduced as toxicity and retained normal histological architecture of renal tissue, inhibited captase-3 mediated tubular cell apoptosis NAD, PH NF- $\mathrm{KB}$ and increases the expression of Nrf2 in renal tissue. There are also some experiments about decreasing of nephrotoxicity of iron by silymarin [23, 24], and experiments were performed with rats. Such renal iron overload in the body is observed in thalassemia (25). Therefore, silymarin may also be useful in the treatment of this disease.

However, in discussing the detoxicating effect of silymarin has to be borne in mind that the compounds contained in the extract, may form complex compounds with heavy metals (chelating properties).

\section{Others}

Nabavi et al. (26) investigated the effect of silymarin against sodium fluoride intoxication (NaF). Nephrotoxicity was induced by 1 week exposure to $\mathrm{NaF} 600 \mathrm{ppm}$ in drinking water. Thiobarbituric acid reactive substance levels, reduced glutathione, as well as superoxide dismutase and catalase activities in renal tissue homogenates were evaluated. Serum biochemical markers of renal impairment, including creatinine, serum urea, blood urease nitrogen, uric acid levels, as well as phosphate and calcium levels, were also evaluated. Intoxication with $\mathrm{NaF}$ caused a significant increase in the levels of thiobarbituric acid reactive substances ( $46 \%$ relative to control) 
and decreased the concentration of glutathione $(47 \%)$ and the activity of superoxide dismutase $(46 \%)$ and catalase $(41 \%)$ in renal tissue homogenates. Intoxication with $\mathrm{NaF}$ also causes significant changes in the biochemical markers of the kidney, increasing levels of urea, uric acid, urea, blood nitrogen, creatinine and phosphate, and decreasing calcium levels. Silymarin, used as a standard antioxidant, also shows nephroprotective activity. Silymarin reduces the renal toxicity of bromobenzene (27).

Recent findings from animal experiments suggest a moderate association between silymarin and the reduced risk of contrastinduced nephropathy (28). There is similar evidence from clinical investigations $(29,30)$. To elucidate the underlying mechanism of high glucose cytotoxicity and the cytotoxic properties of antioxidants against metabolic disorders Wenzel et al. (31) conducted a study in human mesangial cell (HMC) cultures. The 8-day incubation of high glucose HMC (30 $\mathrm{mM}$ ) results in extracellular accumulation of the matrix protein fibronectin (FN). Glucoseinduced changes in FN are not due to osmotic effects, as assessed by iso-osmotic mannitol control. Rather, they are mediated by free oxygen radicals because the combined treatment of HMC with high glucose concentration and the antioxidant flavonoid silibinin (given as a water-soluble derivative of silybinin-C-2,3-dihydrogen succinate disodium salt) prevents the accumulation of FN. This is further confirmed by the determination of maladondialdehyde, a product of lipid peroxidation. Incubation of HMC with high glucose results in an increase in malonicdialdehyde in cellular homogenates, which is completely counteracted by silybinin. Silibinin alone has no effect on protein synthesis and culture growth. The data presented are compatible with the oxidative stress induced by the high glucose concentration in HMC cultures and the role of silybinin in improving glucose cytotoxicity in renal cells.

\section{CONCLUSIONS}

The use of silymarin can reduce the nephrotoxicity of a number of compoundsfungal poisons, organic solvents, heavy metal compounds, and the like. These silymarin properties should be studied in greater detail and put into practice.

\section{REFERENCES}

1. Dashti-Khavidaki S., Shahbazi F., Khalili H., Lessan-Pezeshki M. Potential renoprotective effects of silymarin against nephrotoxic drugs: a review of literature. $J$ Pharm Pharm Sci., 15 (1), 112, Review, (2012).

2. Zholobenko A., Modriansky M. Silymarin and its constituents in cardiac preconditioning, Fitoterapia, 97, 122 (2014) Sep. doi: 10.1016/j.fitote.2014.05.016.Epub 2014 May 29, Review.

3. Jung H., Lee D. Synergistic antibacterial effect between silybin and N,N'dicyclohexylcarbodiimide in clinical Pseudomonas aeruginosa isolates. $J$ Microbiol, 2008 Aug;46(4):462-7. doi: 10.1007/s12275-008-0138-7. Epub 2008 Aug 31.

4. Delmas D, Xiao J., Vejux A., Aires V. Silymarin and Cancer: A Dual Strategy in Both in Chemoprevention and Chemosensitivity. Molecules. 2020 Apr $25 ; 25$

(9):2009. doi:

10.3390/molecules25092009.

5. Sanhueza J., Valdes J., Campos R., Garrido A., Valenzuela A. Changes in the xanthine dehydrogenase/xanthine oxidase ratio in the rat kidney subjected to ischemiareperfusion stress: preventive effect of some flavonoids. Res Commun Chem Pathol Pharmacol., 78 (2), 211 (1992), Nov.

6. Ivanov V., Slavova V., Georgieva D., Petrova-Tacheva V., Tolekova A. Use of silymarin for reducing nephrotoxicity caused by medicaments. Bulgarian chemical communications. Special Issue D, vol. 52, 2020, pp 136-141

7. Enjalbert F., Rapior S., Nouguier-Soulé J., Guillon S., Amouroux N., Cabot C. Treatment of amatoxin poisoning: 20-year retrospective analysis. $J$ Toxicol Clin Toxicol., 40 (6), 715 Review (2002).

8. Vogel G., Braatz R., Mengs U. On the nephrotoxicity of alpha-amanitin and the antagonistic effects of silymarin in rats. Agents Actions, 9 (2), 221 (1979), Jun.

9. Rastogi R., Srivastava A., Rastogi A. Long term effect of aflatoxin $\mathrm{B}_{1}$ on lipid peroxidation in rat liver and kidney: effect of picroliv and silymarin, Phytother Res., 15(4), 307 (2001) Jun.

10.Trakulsrichai S., Sriapha C., Tongpoo A., Udomsubpayakul U., Wongvisavakorn S., 
Srisuma S., Wananukul W. Clinical characteristics and outcome of toxicity from Amanita mushroom poisoning. Int $J$ Gen Med., 10, 395 (2017) Nov 3. doi: 10.2147/IJGM.S141111. eCollection 2017.

11.Svendsen B., Gjellestad A., Eivindson G., Berentsen G., Jacobsen D. Serious mushroom poisoning by Cortinarius and Amanita virosa,Tidsskr Nor LaegeforenNorwegian, 122 (8), 777 (2002) Mar 20.

12.He Q., Riley R., Sharma R. Pharmacological antagonism of fumonisin B1 cytotoxicity in porcine renal epithelial cells (LLC-PK1): a model for reducing fumonisin-induced nephrotoxicity in vivo. Pharmacol Toxicol., 90 (5), 268 (2002) May.

13.Abdel-Kader M., Abulhamd A., Hamad A., Alanazi A., Ali R., Alqasoumi S. Evaluation of the hepatoprotective effect of combination between hinokiflavone and Glycyrrhizin against $\mathrm{CCl}_{4}$ induced toxicity in rats. Saudi Pharm J., 26(4), 496 (2018) May. doi: 10.1016/j.jsps.2018.02.009. Epub 2018 Feb 11.

14. Younis T., Rasul A., Jabeen F., Hussain G., Altaf J., Jafri L., Rani R., Khan M., Sarfraz I., Ali M. Ameliorating role of methanolic leaves extract of Fraxinusxanthoxyloides against CCl4-challanged nephrotoxicity in rats. Pak $J$ Pharm Sci., $31(4$ (Supplementary)), 1475 (2018) Jul.

15.Ghosh S., Sarkar A., Bhattacharyya S., Sil P. Silymarin Protects Mouse Liver and Kidney from Thioacetamide Induced Toxicity by Scavenging Reactive Oxygen Species and Activating PI3K-Akt Pathway, Front Pharmacol., 7, 481 (2016) Dec 15. doi: 10.3389/fphar.2016.00481. eCollection 2016.

16.Cengiz M. Renoprotective effects of Silybum marianum (L.) Gaertn (Silymarin) on thioacetamide-induced renal injury: Biochemical and histopathological approach. Pak J Pharm Sci., $31 \quad$ (5 (Supplementary)), 2137 (2018), Sep.

17.Tunca R., Sozmen M., Citil M., Karapehlivan M., Erginsoy S., Yapar K. Pyridine induction of cytochrome P450 $1 \mathrm{~A} 1$, iNOS and metallothionein in Syrian hamsters and protective effects of silymarin. Exp Toxicol Pathol., 61(3),243 (2009) May. doi: 10.1016/j.etp.2008.05.011. Epub 2009 Apr 1.
18.Alcaraz-Contreras Y., Mendoza-Lozano R., Martínez-Alcaraz E., Martínez-Alfaro M., Gallegos-Corona M., Ramírez-Morales M., Vázquez-Guevara M. Silymarin and dimercaptosuccinic acid ameliorate leadinduced nephrotoxicity and genotoxicity in rats, Hum ExpToxicol., 35(4), 398 (2016) Apr. doi: 10.1177/0960327115591373. Epub 2015 Jun 15.

19.El-Khishin I., El-Fakharany Y., Hamid O. Role of garlic extract and silymarin compared to dimercaptosuccinic acid (DMSA) in treatment of lead induced nephropathy in adult male albino rats. Toxicol Rep., 2, 824 (2015) May 8. doi: 10.1016/j.toxrep.2015.04.004. eCollection 2015.

20.Chtourou Y., Garoui el M., Boudawara T., Zeghal N. Protective role of silymarin against manganese-induced nephrotoxicity and oxidative stress in rat, Environ Toxicol, 29(10), 1147 (2014) Oct. doi: 10.1002/tox.21845. Epub 2013 Jan 22.

21.Navaneethan D., Rasool M. p-Coumaric acid, a common dietary polyphenol, protects cadmium chloride-induced nephrotoxicity in rats,Ren Fail., 36 (2), 244 (2014) Mar. doi: 10.3109/0886022X.2013.835268. Epub 2013 Sep 24.

22.Prabu S., Muthumani M. Silibinin ameliorates arsenic induced nephrotoxicity by abrogation of oxidative stress, inflammation and apoptosis in rats. $\mathrm{Mol}$ Biol Rep., 39 (12), 11201 (2012), Dec. doi: 10.1007/s11033-012-2029-6. Epub 2012 Oct 16.

23.Valenzuela A., Aspillaga M., Vial S., Guerra R. Selectivity of silymarin on the increase of the glutathione content in different tissues of the rat, Planta Med., 55 (5), 420 (1989) Oct.

24.Navidi-Shishaone M., Mohhebi S., Nematbakhsh M., Roozbehani S., Talebi A., Pezeshki Z., Eshraghi-Jazi F., Mazaheri S., Shirdavani S., Gharagozloo M., Moaeidi B. Co-Administration of Silymarin and Deferoxamine against Kidney, Liver and Heart Iron Deposition in Male Iron Overload Rat Model. Int J Prev Med., 5 (1), 110 (2014) Jan.

25.Demosthenous Ch. , Vlachaki E., Apostolou Ch., Eleftheriou P., Kotsiafti Agg., Vetsiou Ev., Mandala Evd., Perifanis D., Sarafidis P. Beta-thalassemia: renal complications and mechanisms: a narrative 
review. Hematology Volume 24, 2019, Isue 1 Pages 426-438, Published online: 04 Apr 2019

26.Nabavi S., Habtemariam S. Nabavi S., Sureda A., Daglia M., Moghaddam A., Amani, M. Protective effect of gallic acid isolated from Peltiphyllumpeltatum against sodium fluoride-induced oxidative stress in rat's kidney. Mol Cell Biochem., 372 (1-2), 233 (2013) Jan. doi: 10.1007/s11010-0121464-y. Epub 2012 Sep 27.

27.Vedi M., Rasool M., Sabina E.P. Protective effect of administration of Withaniasomifera against bromobenzene induced nephrotoxicity and mitochondrial oxidative stress in rats. Ren Fail., 36 (7), 1095 (2014) Aug. doi: 10.3109/0886022X.2014.918812. Epub 2014 May 21.

28.Santos V., Peters B., Côco L. Alves G., Assis Ar., Nogueira Br., Meyrelles S., Porto M., Vasquez El., Campagnaro B., Pereira Th. Silymarin protects against radiocontrast-induced nephropathy in mice.
Life Sciences. Volume 228, 1 July 2019, Pages 305-315

29. Sedighifard Z., Roghani F., Bidram P., Harandi S.A., Molavi S. Silymarin for the Prevention of ContrastInduced Nephropathy: A PlaceboControlled Clinical Trial. Int J Prev Med. 2016 Jan 22;7:23. doi: 10.4103/20087802.174762 .

eCollection 2016.PMID: 26941924

30.Kuo Y., Chang H., Chang Y., Wu H., Chen C.. Evaluation of nephroprotection of silymarin on contrast-

induced nephropathy in liver cirrhosis patients: A population-based cohort study. Medicine (Baltimore). 2018 Sep;97(37):e12243. doi: 10.1097/MD.0000000000012243.PMID: 30 212956

31.Wenzel S., Stolte H., Soose M. Effects of silibinin and antioxidants on high glucoseinduced alterations of fibronectin turnover in human mesangial cell cultures. $J$ Pharmacol Exp Ther., 279 (3), 1520 (1996) Dec. 